\title{
AN INITIAL VALUE PROBLEM FOR TWO-DIMENSIONAL IDEAL INCOMPRESSIBLE FLUIDS WITH CONTINUOUS VORTICITY
}

\author{
Elaine Cozzi
}

\begin{abstract}
We study an initial value problem for the two-dimensional Euler equation. In particular, we consider the case where initial data belongs to a critical or subcritical Besov space, and initial vorticity is continuous with compact support. Under these assumptions, we conclude that the solution to the Euler equation loses an arbitrarily small amount of regularity as time evolves.
\end{abstract}

\section{Introduction}

In this paper, we study the Euler Equation modeling incompressible fluid flow on $\mathbb{R}^{2}$, given by

$$
\begin{aligned}
& \partial_{t} v(t, x)=-v \cdot \nabla v-\nabla p, \\
& \nabla \cdot v=0, \\
& \left.v\right|_{t=0}=v_{0},
\end{aligned}
$$

where the vorticity of the fluid is given by

$$
\omega=\omega(v)=\partial_{1} v^{2}-\partial_{2} v^{1} .
$$

It is known that when initial data for (1) is in the supercritical Sobolev space $W^{s+1, p}\left(\mathbb{R}^{2}\right)$, where $s p>2$, the solution does not lose any regularity as time evolves (see, for example, [4], [5]). Much less is known when initial data belongs to the critical Sobolev spaces, where $s p=2$, or to the subcritical Sobolev spaces, where $s p<2$. We therefore restrict our attention to these two cases. In addition, we assume that the initial vorticity is continuous with compact support.

The motivation for this paper is a result of Bahouri and Chemin found in [1], where the authors show that a lower bound for the Sobolev exponent of $\omega(t)$ is determined by the log-Lipschitz norm of $v(t)$. They define

$$
V(t)=\sup _{|x-y| \leq 1} \frac{|v(t, x)-v(t, y)|}{|x-y|(1-\log |x-y|)},
$$

and they prove the following theorem:

Theorem 1. Let $v$ be a solution to (1) such that $\omega\left(v_{0}\right)=\omega_{0} \in L^{\infty}\left(\mathbb{R}^{2}\right) \cap W^{s, p}\left(\mathbb{R}^{2}\right)$, for $s p \leq 2$ and $s \in(0,1]$. Fix $s^{\prime}<s$, and define $\sigma\left(s^{\prime}, t\right)=s^{\prime} \exp \left(-\int_{0}^{t} V(\tau) d \tau\right)$. Then $\omega(t) \in W^{\sigma\left(s^{\prime}, t\right), p}\left(\mathbb{R}^{2}\right)$ for all $t \in \mathbb{R}$.

Received by the editors June 20, 2006. 
In this paper, we show that, if we also assume $\omega_{0}$ is continuous with compact support, then we can improve the lower bound for loss of regularity to an arbitrarily small amount. More precisely, we show that, given $\epsilon>0$ arbitrarily small, and $T>0$ fixed, if $\omega_{0} \in W^{s, p}\left(\mathbb{R}^{2}\right) \cap C_{c}\left(\mathbb{R}^{2}\right)$ with $s p \leq 2, p \in(1, \infty)$, and $s \in(0,2)$, then $\omega(t)$ belongs to $W^{s-\epsilon, p}\left(\mathbb{R}^{2}\right)$ for all $t \in[0, T]$. As in [1], we study the vorticity equation corresponding to the Euler equation. When $n=2$, the vorticity equation is given by

$$
\begin{array}{r}
\partial_{t} \omega+v \cdot \nabla \omega=0, \\
\left.\omega\right|_{t=0}=\omega_{0} .
\end{array}
$$

To prove our result, we show that $\omega(t)$ belongs to the Besov space $B_{p, \infty}^{s-\epsilon}$ (see Definition 3 ) for all $t$ in a finite time interval $[0, T]$. Our general approach is to localize the frequency of the terms of (2), which results in a new equation with a commutator term on the right-hand side:

$$
\begin{aligned}
& \partial_{t} \Delta_{q} \omega+v \cdot \nabla \Delta_{q} \omega=\left[v \cdot \nabla, \Delta_{q}\right] \omega, \\
& \left.\Delta_{q} \omega\right|_{t=0}=\Delta_{q} \omega_{0} .
\end{aligned}
$$

We then prove the necessary estimate on the $L^{p}$ norm of the commutator on the right-hand side of (3), and apply a Gronwall argument to show that $\omega(t)$ is in $B_{p, \infty}^{s-\epsilon}$.

The main novelty of this paper is that our methods allow us to draw conclusions for $\omega_{0} \in B_{p, \infty}^{s}\left(\mathbb{R}^{2}\right) \cap C_{c}\left(\mathbb{R}^{2}\right)$ for all $s \in(0,2)$, whereas, if we restrict our attention to the case $s \in(0,1]$, we can prove the result by combining methods used in [1] with the following theorem, which we prove in Section 3:

Theorem 2. Let $v$ be a solution to (1) such that $\omega\left(v_{0}\right)=\omega_{0} \in C_{c}\left(\mathbb{R}^{2}\right)$. Let $g(t)$ be the measure-preserving homeomorphism in $\mathbb{R}^{2}$ satisfying $\partial_{t} g(t, x)=v(t, g(t, x))$. Given $\delta>0$ and $T>0$, it follows that $\left\|g(t)^{-1}-I d\right\|_{C^{1-\delta}} \in L^{\infty}([0, T])$.

\section{Littlewood-Paley Decomposition and Function Spaces}

In this section, we set notation and recall the definitions of the function spaces which we use throughout the proof of our main theorem.

Proposition 1. There exists two radial functions $\chi \in S$ and $\varphi \in S$ satisfying the following properties:

(i) $\operatorname{supp} \chi \subset\left\{\xi \in \mathbb{R}^{2}: 0 \leq|\xi| \leq \frac{4}{3}\right\}$,

(ii) $\operatorname{supp} \varphi \subset\left\{\xi \in \mathbb{R}^{2}: \frac{3}{4} \leq|\xi| \leq \frac{8}{3}\right\}$,

(iii) $\chi(\xi)+\sum_{j=0}^{\infty} \varphi_{j}(\xi)=1$,

where $\varphi_{j}(\xi)=\varphi\left(2^{-j} \xi\right)\left(\right.$ so $\left.\check{\varphi}_{j}(x)=2^{j n} \check{\varphi}\left(2^{j} x\right)\right)$.

Proof. See [6].

Observe that, if $\left|j-j^{\prime}\right| \geq 2$, then $\operatorname{supp} \varphi_{j} \cap \operatorname{supp} \varphi_{j^{\prime}}=\emptyset$, and, if $j \geq 1$, then supp $\varphi_{j} \cap \operatorname{supp} \chi=\emptyset$.

Definition 1. Let $f \in S^{\prime}$. We define

$\Delta_{-1} f=\chi(D) f=\check{\chi} * f$. 
For $j \geq 0, \Delta_{j} f=\check{\varphi}_{j} * f$.

For $j \leq-2, \Delta_{j} f=0$.

For $k \in \mathbb{Z}, S_{k} f=\sum_{j=-1}^{k-1} \Delta_{j} f=\chi\left(2^{-k} D\right) f$.

Definition 2. Let $s \in \mathbb{R}$. We define the Zygmund space $C_{*}^{s}$ to be the space of tempered distributions $f$ such that

$$
\|f\|_{C_{*}^{s}}:=\sup _{q \geq-1} 2^{q s}\left\|\Delta_{q} f\right\|_{L^{\infty}}<\infty .
$$

It is well known that the norm on $C_{*}^{s}$ is equivalent to the classical $C^{s}$ norm when $s$ is not an integer and $s>0$. For a proof of this, see [3], Proposition 2.3.1.

Definition 3. Let $s \in \mathbb{R}, p, q \in[1, \infty]$. We define the inhomogeneous Besov space $B_{p, q}^{s}$ to be the space of tempered distributions $f$ such that

When $q=\infty$, write

$$
\|f\|_{B_{p, q}^{s}}:=\left(\sum_{j=-1}^{\infty} 2^{j q s}\left\|\Delta_{j} f\right\|_{L^{p}}^{q}\right)^{\frac{1}{q}}<\infty .
$$

$$
\|f\|_{B_{p, \infty}^{s}}:=\sup _{j \geq-1} 2^{j s}\left\|\Delta_{j} f\right\|_{L^{p}} .
$$

Remark. Note that $B_{\infty, \infty}^{s}=C_{*}^{s}$.

Definition 4. The space of log-Lipschitz functions, denoted by $L L$, is the space of bounded functions $f$ on $\mathbb{R}^{n}$ such that

$$
\|f\|_{L L}:=\|f\|_{L^{\infty}}+\sup _{|x-y| \leq 1} \frac{|f(x)-f(y)|}{|x-y|(1-\log |x-y|)}<\infty .
$$

An important tool throughout the proof of the main theorem will be a decomposition introduced by J.-M. Bony in [2]. We recall the definition of the paraproduct and remainder used in this decomposition.

Definition 5. Define the paraproduct of two functions $f$ and $g$ by

$$
T_{f} g=\sum_{\substack{i, j \\ i \leq j-2}} \Delta_{i} f \Delta_{j} g=\sum_{j=1}^{\infty} S_{j-1} f \Delta_{j} g .
$$

We use $R(f, g)$ to denote the remainder. $R(f, g)$ is given by the following bilinear operator:

$$
R(f, g)=\sum_{\substack{i, j \\|i-j| \leq 1}} \Delta_{i} f \Delta_{j} g .
$$

Bony's decomposition gives

$$
f g=T_{f} g+T_{g} f+R(f, g) .
$$




\section{Proof of Theorem 2}

It is known (see [10]) that if $\|v(t)\|_{L L} \in L_{\text {loc }}^{1}\left(\mathbb{R}^{+}\right)$, then the flow $g(t)$ exists, is unique, is a measure-preserving homeomorphism from $\mathbb{R}^{2}$ to $\mathbb{R}^{2}$, and satisfies the equation

$$
g(t, x)=x+\int_{0}^{t} v(\tau, g(\tau, x)) d \tau
$$

Furthermore,

$$
g(t)-I d \in C^{\exp \left(-\int_{0}^{t}\|v(\tau)\|_{L L} d \tau\right)} .
$$

From (5), we see that the Holder exponent of $g(t)-I d$ is determined by the logLipschitz norm of $v$. One can characterize log-Lipschitz functions using the following inequality (see [1]):

$$
C^{-1}\|f\|_{L L} \leq\left\|S_{0} f\right\|_{L^{\infty}}+\sup _{q \geq 1} \frac{\left\|\nabla S_{q} f\right\|_{L^{\infty}}}{q+1} \leq C\|f\|_{L L}
$$

for a constant $C>0$. When computing the Holder exponent of $g(t)-I d,(5)$ and (6) motivate us to study the behavior of the quantity $\left\|\nabla S_{q} v(t)\right\|_{L^{\infty}}$. In this section, we assume $\omega_{0} \in C_{c}\left(\mathbb{R}^{2}\right)$, and we show that, given $\epsilon>0$ and $T>0,\left\|\nabla S_{q} v(t)\right\|_{L^{\infty}} \leq$ $\epsilon(q+1)$ for sufficiently large $q$ and for all $t \in[0, T]$. We then conclude that $g(t)$ is locally Holder continuous with Holder exponent arbitrarily close to 1 . We begin with the following lemma:

Lemma 2. Let $u \in C_{c}\left(\mathbb{R}^{2}\right)$. Given $\epsilon>0$, there exists an $N>0$ such that $\left\|\Delta_{j} u\right\|_{L^{\infty}}<$ $\epsilon$ for all $j>N$.

Proof. Since $u \in C_{c}\left(\mathbb{R}^{2}\right)$, we can construct a sequence $\left(u_{k}\right) \subset C_{c}^{\infty}\left(\mathbb{R}^{2}\right)$ such that $u_{k} \rightarrow u$ uniformly. Also, since $u_{k} \in C_{c}^{\infty}\left(\mathbb{R}^{2}\right)$, there exists an $N>0$ so that $\left\|\Delta_{j} u_{k}\right\|_{L^{\infty}}<\frac{\epsilon}{2}$ for $j>N$. Therefore, for $k$ sufficiently large, and for all $j>N$, we have

$$
\begin{aligned}
\left\|\Delta_{j} u\right\|_{L^{\infty}} & \leq\left\|\Delta_{j}\left(u_{k}-u\right)\right\|_{L^{\infty}}+\left\|\Delta_{j} u_{k}\right\|_{L^{\infty}} \\
& <\epsilon .
\end{aligned}
$$

This completes the proof.

In dimension two, we can rewrite the vorticity equation given in $(2)$ as $\omega(t, x)=$ $\omega_{0}\left(g(t)^{-1}(x)\right)$. Therefore, if we assume that $\omega_{0} \in C_{c}$, then $\omega(t) \in C_{c}$ for all $t \in \mathbb{R}$. We now apply Lemma 2 to $\omega(t)$ and conclude that, for fixed $t$, given $\epsilon>0$, there exists $N_{t}$ such that $\sup _{j>N_{t}}\left\|\Delta_{j} \omega(t)\right\|_{L^{\infty}}<\epsilon$. In what follows, we need $N_{t}$ to be time independent. We therefore prove the following lemma:

Lemma 3. Let $v$ be a solution to (1) such that $\omega\left(v_{0}\right)=\omega_{0} \in C_{c}\left(\mathbb{R}^{2}\right)$. Given $\epsilon>0$ and $T>0$, there exists an $N=N(T, \epsilon)$ so that $\sup _{j>N}\left\|\Delta_{j} \omega(t)\right\|_{L^{\infty}}<\epsilon$ for all $t \in[0, T]$.

Proof. To prove the lemma, we use the Ascoli-Arzela Theorem to show that the set $A=\{\omega(t): t \in[0, T]\}$ is compact in $C(M)$, where $M$ is the compact subset of $\mathbb{R}^{2}$ containing the supports of all elements of $A$. The set $A$ is clearly equibounded as 
$\|\omega(t)\|_{L^{\infty}}=\left\|\omega_{0}\right\|_{L^{\infty}}$ for all $t \in[0, T]$. To show equicontinuity, we observe that, for $x_{1}$ and $x_{2}$ in $\mathbb{R}^{2}$ satisfying $g\left(t_{1}, x_{1}\right)=g\left(t_{2}, x_{2}\right)=x$, we have,

$$
\begin{aligned}
\left|x_{1}-x_{2}\right| & \leq\left|t_{1}-t_{2}\right| \sup _{\tau \in\left[t_{1}, t_{2}\right]}\|v(\tau)\|_{L^{\infty}} \\
& \leq C\left|t_{1}-t_{2}\right|\left(\left\|\omega_{0}\right\|_{L^{p}}+\left\|\omega_{0}\right\|_{L^{\infty}}\right),
\end{aligned}
$$

where we used the bound $\|v(t)\|_{L^{\infty}} \leq C\left(\left\|\omega_{0}\right\|_{L^{p}}+\left\|\omega_{0}\right\|_{L^{\infty}}\right)$ for $p \in(1,2)$. (For a proof of this bound, we refer the reader to [8], Theorem 3.1.) Therefore, by uniform continuity of $\omega_{0}$, given $\epsilon>0$, there exists $\delta>0$ such that, for $\left|t_{1}-t_{2}\right|<\delta$ and for all $x \in \mathbb{R}^{2}$,

$$
\begin{aligned}
\left|\omega\left(t_{1}, x\right)-\omega\left(t_{2}, x\right)\right| & =\left|\omega_{0}\left(x_{1}\right)-\omega_{0}\left(x_{2}\right)\right| \\
& <\frac{\epsilon}{2} .
\end{aligned}
$$

We conclude that, for $\left|t_{1}-t_{2}\right|<\delta$,

$$
\begin{aligned}
& || \omega\left(t_{1}, x_{1}\right)-\omega\left(t_{1}, x_{2}\right)|-| \omega\left(t_{2}, x_{1}\right)-\omega\left(t_{2}, x_{2}\right)|| \\
& \leq\left|\omega\left(t_{1}, x_{1}\right)-\omega\left(t_{2}, x_{1}\right)\right|+\left|\omega\left(t_{1}, x_{2}\right)-\omega\left(t_{2}, x_{2}\right)\right|<\epsilon .
\end{aligned}
$$

Equicontinuity follows from (7). Finally, we observe that $A$ is closed in $C(M)$ by sequential continuity of $\omega$ in time and compactness of $[0, T]$. We conclude that $A$ is compact in $C(M)$. From this compactness, it follows that for any $t \in[0, T]$ and $\epsilon>0$, there exists $t_{i} \in[0, T], i=1,2, \ldots, M$, such that $\left\|\Delta_{j} \omega(t)\right\|_{L^{\infty}}<\frac{\epsilon}{2}+\left\|\Delta_{j} \omega\left(t_{i}\right)\right\|_{L^{\infty}}$ for all $j$. Furthermore, given $\omega\left(t_{i}\right)$, there exists an $N_{t_{i}}$ such that $\sup _{j>N_{t_{i}}}\left\|\Delta_{j} \omega\left(t_{i}\right)\right\|_{L^{\infty}}<$ $\frac{\epsilon}{2}$. Let $N=\max \left\{N_{t_{1}}, \ldots . N_{t_{M}}\right\}$. Then, for all $t \in[0, T]$,

$$
\sup _{j>N}\left\|\Delta_{j} \omega(t)\right\|_{L^{\infty}}<\epsilon .
$$

This completes the proof.

We bound $\left\|\Delta_{j} \nabla v\right\|_{L^{\infty}}$ by $C\left\|\Delta_{j} \omega\right\|_{L^{\infty}}$ if $j \geq 0$, and we bound $\left\|\Delta_{-1} \nabla v(t)\right\|_{L^{\infty}}$ with $C|| \omega_{0} \|_{L^{p}}$ for $p \in(1, \infty)$ using Bernstein's inequality. From Lemma 3, we conclude that, for $N$ sufficiently large, and for all $t \in[0, T]$,

$$
\left\|S_{N} \nabla v(t)\right\|_{L^{\infty}} \leq \epsilon(N+1) .
$$

We now use (8) to compute the Holder exponent for the flow corresponding to the velocity of a fluid with initial vorticity in $C_{c}\left(\mathbb{R}^{2}\right)$, which will complete the proof of Theorem 2. We show that, given $\epsilon>0$ and $T>0, g(t)^{-1}-I d$ belongs to $C^{\sigma(t)}\left(\mathbb{R}^{2}\right)$ for all $t \in[0, T]$, where $\sigma(t)=e^{-C t \epsilon}$, and $C$ is an absolute constant. We then let $\delta=1-e^{-C T \epsilon}$, and we make $\delta$ as small as we would like by our choice of $\epsilon$.

Fix $\epsilon>0$. Write $v=v_{1, N}+v_{2, N}$, where $v_{1, N}=S_{N-1} v$, and $v_{2, N}=\left(I d-S_{N-1}\right) v$. By (8), it follows that $\left|v_{1, N}(t, x)-v_{1, N}(t, y)\right| \leq|| \nabla S_{N-1} v(t) \|_{L^{\infty}}|x-y| \leq C N \epsilon \mid x-$ $y \mid$ for large enough $N$. Similarly, for large $N$ we can conclude that $\mid v_{2, N}(t, x)-$ $v_{2, N}(t, y) \mid \leq C \sum_{j=N-1}^{\infty} 2^{-j}\left\|\Delta_{j} \nabla v(t)\right\|_{L^{\infty}} \leq C 2^{-N} \epsilon$. Letting $N=-\log _{2}|x-y|$, we have that for $|x-y|$ sufficiently small,

$$
\begin{aligned}
|v(t, x)-v(t, y)| & \leq\left|\left(v_{1, N}(t, x)+v_{2, N}(t, x)\right)-\left(v_{1, N}(t, y)+v_{2, N}(t, y)\right)\right| \\
& \leq C\left(\epsilon\left(-\log _{2}|x-y|\right)|x-y|+\epsilon|x-y|\right) \\
& \leq C \epsilon\left(1-\log _{2}|x-y|\right)|x-y|
\end{aligned}
$$


We now use (9) and Osgood's Lemma (see [3], Lemma 5.2.1) to compute properties of the flow. We write

$$
|v(t, g(t, x))-v(t, g(t, y))| \leq C \epsilon\left(1-\log _{2}|g(t, x)-g(t, y)|\right)|g(t, x)-g(t, y)|
$$

whenever $|g(t, x)-g(t, y)|<\delta$. From (4), this gives

$$
|g(t, x)-g(t, y)| \leq|x-y|+\int_{0}^{t} C \epsilon\left(1-\log _{2}|g(\tau, x)-g(\tau, y)|\right)|g(\tau, x)-g(\tau, y)| d \tau \text {. }
$$

By Osgood's Lemma, we conclude that

$$
-\log (1-\log |g(t, x)-g(t, y)|)+\log (1-\log |x-y|) \leq C t \epsilon .
$$

Taking the exponential twice, we get

$$
\frac{|g(t, x)-g(t, y)|}{|x-y|^{-C t \epsilon}} \leq e^{1-e^{-C t \epsilon}} \leq e
$$

whenever $|x-y|<\delta$, which gives

$$
\frac{|(g(t, x)-x)-(g(t, y)-y)|}{|x-y|^{-C t \epsilon}} \leq e+1 .
$$

In the case $|x-y| \geq \delta$, we have

$$
\frac{|(g(t, x)-x)-(g(t, y)-y)|}{|x-y|^{-C t \epsilon}} \leq 2 \delta^{-e^{-C t \epsilon}}|| g(t)-\left.I d\right|_{L^{\infty}} .
$$

To see that $\left\|g(t)^{-1}-I d\right\|_{C^{1-\delta}} \in L_{l o c}^{\infty}\left(\mathbb{R}^{+}\right)$, we observe that

$$
\begin{aligned}
\sup _{x, y \in \mathbb{R}^{2}} \frac{|(g(t, x)-x)-(g(t, y)-y)|}{|x-y|^{e^{-C t \epsilon}}} & \leq(e+1)+2 \delta^{-e^{-C t \epsilon}}\left\|\int_{0}^{t} v(\tau, g(\tau, \cdot)) d \tau\right\|_{L^{\infty}} \\
& \leq(e+1)+2 \delta^{-1} T\left(\left.\left\|\omega_{0}\right\|\right|_{L^{p}}+\left\|\omega_{0}\right\|_{L^{\infty}}\right)
\end{aligned}
$$

for all $t \in[0, T]$. This completes the proof of Theorem 2 .

\section{Paradifferential Estimates for the Transport Equation}

In this section, we consider an initial value problem for the vorticity equation corresponding to the two-dimensional Euler Equation. When $n=2$, the vorticity equation is given by

$$
\begin{array}{r}
\partial_{t} \omega+v \cdot \nabla \omega=0, \\
\left.\omega\right|_{t=0}=\omega_{0} .
\end{array}
$$

Note that, if $\omega$ satisfies (10), then $\Delta_{q} \omega$ satisfies the following equation:

$$
\begin{aligned}
& \partial_{t} \Delta_{q} \omega+v \cdot \nabla \Delta_{q} \omega=\left[v \cdot \nabla, \Delta_{q}\right] \omega, \\
& \left.\Delta_{q} \omega\right|_{t=0}=\Delta_{q} \omega_{0} .
\end{aligned}
$$

We want to prove the following estimate:

Proposition 4. Let $p \in(1, \infty)$ and $\sigma>0$ be fixed. Then there exists two positive constants $C_{1}(\sigma)$ and $C_{2}$ such that

$$
\left\|\left[v \cdot \nabla, \Delta_{q}\right] \omega\right\|_{L^{p}} \leq C_{1}(\sigma)\left(C_{2}+\left\|S_{q-1} \nabla v\right\|_{L^{\infty}}\right) 2^{-q \sigma}\|\omega\|_{B_{p, \infty}^{\sigma}} .
$$


Proof. We consider the cases $q \geq 4$ and $q<4$ separately. We first assume $q \geq 4$ and use Bony's decomposition to write

$$
\left[v \cdot \nabla, \Delta_{q}\right] \omega=\sum_{j=1}^{2}\left[T_{v_{j}} \partial_{j}, \Delta_{q}\right] \omega+\left[T_{\partial_{j}} \cdot v_{j}, \Delta_{q}\right] \omega+\left[\partial_{j} R\left(v_{j}, \cdot\right), \Delta_{q}\right] \omega .
$$

We address each piece of the sum separately. We start with $\left[T_{v_{j}} \partial_{j}, \Delta_{q}\right] \omega$. Write

$$
\left[T_{v_{j}} \partial_{j}, \Delta_{q}\right] \omega=\sum_{q^{\prime}=q-4}^{q+4}\left[S_{q^{\prime}-1}\left(v_{j}\right), \Delta_{q}\right] \Delta_{q^{\prime}} \partial_{j} \omega .
$$

Letting $u=\Delta_{q^{\prime}} \partial_{j} \omega$, letting $h=\check{\phi}$ (recall we are assuming $q \geq 4$ here), and keeping in mind that $\left|q^{\prime}-q\right| \leq 4$, we have

$$
\begin{aligned}
& \left\|\left[S_{q^{\prime}-1}\left(v_{j}\right), \Delta_{q}\right] u\right\|_{L^{p}}= \\
& \left\|\int_{\mathbb{R}^{2}} h(y)\left(S_{q^{\prime}-1}\left(v_{j}\right)\left(x-2^{-q} y\right)-S_{q^{\prime}-1}\left(v_{j}\right)(x)\right) u\left(x-2^{-q} y\right) d y\right\|_{L^{p}} \\
& \leq C\left\|S_{q^{\prime}-1} \nabla v\right\|_{L^{\infty}} 2^{-q}\|u\|_{L^{p}} \\
& \leq C 2^{4 \sigma}\left\|S_{q^{\prime}-1} \nabla v\right\|_{L^{\infty}} 2^{-q \sigma}\|\omega\|_{B_{p, \infty}^{\sigma}},
\end{aligned}
$$

where we used the fact that $h \in S$ and therefore $z h(z)$ is integrable, as well as Bernstein's inequality. We now sum over $q^{\prime}$ to get

$$
\begin{aligned}
\left\|\left[T_{v_{j}} \partial_{j}, \Delta_{q}\right] \omega\right\|_{L^{p}} & \leq C 2^{4 \sigma} 2^{-q \sigma}\|\omega\|_{B_{p, \infty}^{\sigma}} \sum_{q^{\prime}=q-4}^{q+4}\left\|S_{q^{\prime}-1} \nabla v\right\|_{L^{\infty}} \\
& \leq C 2^{4 \sigma} 2^{-q \sigma}\|\omega\|_{B_{p, \infty}^{\sigma}}\left(\left\|S_{q-1} \nabla v\right\|_{L^{\infty}}+\|\nabla v\|_{C_{*}^{0}}\right) .
\end{aligned}
$$

We now consider $\left[T_{\partial_{j}} \cdot v_{j}, \Delta_{q}\right] \omega$. To bound $\left\|T_{\partial_{j} \Delta_{q} \omega} v_{j}\right\|_{L^{p}}$, we use Bernstein's inequality and our assumption that $q \geq 4$, as well as properties of our partition of unity, to write

$$
\begin{aligned}
\left\|T_{\partial_{j} \Delta_{q} \omega} v_{j}\right\|_{L^{p}} & \leq \sum_{q^{\prime}=q}^{\infty} C 2^{q} 2^{-q^{\prime}}\left\|S_{q^{\prime}-1} \Delta_{q} \omega\right\|_{L^{p}}\left\|\Delta_{q^{\prime}} \nabla v\right\|_{L^{\infty}} \\
& \leq C\left\|\Delta_{q} \omega\right\|_{L^{p}} \sup _{q^{\prime} \geq q}\left\|\Delta_{q^{\prime}} \nabla v\right\|_{L^{\infty}} \\
& \leq C\|\nabla v\|_{C_{*}^{0}} 2^{-q \sigma}\|\omega\|_{B_{p, \infty}^{\sigma}} .
\end{aligned}
$$

Furthermore, since the Fourier support of $S_{q^{\prime}-1} \partial_{j} \omega \Delta_{q^{\prime}} v_{j}$ is contained in an annulus with inner and outer radius $C_{1} 2^{q^{\prime}}$ and $C_{2} 2^{q^{\prime}}$ respectively, we can write

$$
\begin{aligned}
& \left\|\Delta_{q}\left(T_{\partial_{j} \omega} v_{j}\right)\right\|_{L^{p}} \leq \sum_{q^{\prime}=q-4}^{q+4}\left\|S_{q^{\prime}-1} \partial_{j} \omega\right\|_{L^{\infty}}\left\|\Delta_{q^{\prime}} v\right\|_{L^{p}} \\
& \leq \sum_{q^{\prime}=q-4}^{q+4} C 2^{q^{\prime}} 2^{-q^{\prime}}\left\|S_{q^{\prime}-1} \omega\right\|_{L^{\infty}}\left\|\Delta_{q^{\prime}} \nabla v\right\|_{L^{p}} \\
& \leq \sum_{q^{\prime}=q-4}^{q+4} C\left\|S_{q^{\prime}-1} \nabla v\right\|_{L^{\infty}}\left\|\Delta_{q^{\prime}} \omega\right\|_{L^{p}} \\
& \leq C 2^{4 \sigma}\left(\left\|S_{q-1} \nabla v\right\|_{L^{\infty}}+\|\nabla v\|_{C_{*}^{0}}\right) 2^{-q \sigma}\|\omega\|_{B_{p, \infty}^{\sigma}} .
\end{aligned}
$$


Once again, we used Bernstein's inequality in the second inequality. It is in this inequality that our assumption that $q \geq 4$ is necessary. For the third inequality, we used the fact that Calderon-Zygmund operators are bounded on $L^{p}$ for $p \in(1, \infty)$. Combining (13) and (14), we see that

$$
\left\|\left[T_{\partial_{j}} \cdot v_{j}, \Delta_{q}\right] \omega\right\|_{L^{p}} \leq C 2^{4 \sigma}\left(\left\|S_{q-1} \nabla v_{j}\right\|_{L^{\infty}}+\|\nabla v\|_{C_{*}^{0}}\right) 2^{-q \sigma}\|\omega\|_{B_{p, \infty}^{\sigma}}
$$

for $q \geq 4$.

We now study the remainder term, $\left[\partial_{j} R\left(v_{j}, \cdot\right), \Delta_{q}\right] \omega$. We need the following lemma:

Lemma 5. If $s+\sigma>0$, then $\|R(a, b)\|_{B_{p, \infty}^{s+\sigma}} \leq C(s, \sigma)\|a\|_{B_{\infty, \infty}^{s}}\|b\|_{B_{p, \infty}^{\sigma}}$, where $C(s, \sigma)=C 2^{M \sigma}\left(\frac{1}{1-\left(\frac{1}{2}\right)^{s+\sigma}}+2^{N(s+\sigma)}+\ldots+2^{1(s+\sigma)}\right)$ for fixed positive integers $M$ and $N$.

Proof. For a proof of the lemma, we refer the reader to [9].

To handle the remainder term, we will consider low and high frequencies of $v_{j}$ separately. We begin with $\left[\partial_{j} R\left(\left(I d-\Delta_{-1}\right) v_{j}, \cdot\right), \Delta_{q}\right] \omega$. Using Lemma 5 with $s=$ 1 , Bernstein's inequality, and the fact that the Fourier transform of $\left(I d-\Delta_{-1}\right) \nabla v$ vanishes in a neighborhood of the origin, we write

$$
\begin{aligned}
\left\|\Delta_{q} \partial_{j} R\left(\left(I d-\Delta_{-1}\right) v_{j}, \omega\right)\right\|_{L^{p}} & \leq 2^{-q \sigma}\left\|R\left(\left(I d-\Delta_{-1}\right) v_{j}, \omega\right)\right\|_{B_{p, \infty}^{\sigma+1}} \\
& \leq C(\sigma) 2^{-q \sigma}\left\|\left(I d-\Delta_{-1}\right) v\right\|_{B_{\infty, \infty}^{1}}\|\omega\|_{B_{p, \infty}^{\sigma}} \\
& \leq C(\sigma) 2^{-q \sigma}\|\nabla v\|_{C_{*}^{0}}\|\omega\| \|_{B_{p, \infty}^{\sigma}} .
\end{aligned}
$$

Here $C(\sigma)=C(1, \sigma)$ from Lemma 5 . To bound $\left\|\partial_{j} R\left(\left(I d-\Delta_{-1}\right) v_{j}, \Delta_{q} \omega\right)\right\|_{L^{p}}$, note that

$$
\begin{aligned}
&\left\|\partial_{j} R\left(\left(I d-\Delta_{-1}\right) v_{j}, \Delta_{q} \omega\right)\right\|_{L^{p}} \leq \sum_{\substack{q^{\prime}, q^{\prime \prime} \\
\left|q^{\prime}-q^{\prime \prime}\right| \leq 1 \\
\left|q^{\prime}-q\right| \leq 1}} 2^{q}\left\|\Delta_{q^{\prime \prime}}\left(I d-\Delta_{-1}\right) v\right\|_{L^{\infty}}\left\|\Delta_{q^{\prime}} \Delta_{q} \omega\right\|_{L^{p}} \\
& \leq C|| \nabla v\left\|_{C_{*}^{0}} 2^{-q \sigma}\right\| \omega \|_{B_{p, \infty}^{\sigma}} .
\end{aligned}
$$

In the first inequality above, we used the fact that the support of the Fourier transform of $\Delta_{q^{\prime \prime}}\left(I d-\Delta_{-1}\right) v \Delta_{q^{\prime}} \Delta_{q} \omega$ is contained in a ball with radius $C 2^{q}$, along with Bernstein's inequality, to get the factor $2^{q}$. In the second inequality, we used the inequality $\left\|\left(I d-\Delta_{-1}\right) v\right\|_{B_{\infty, \infty}^{1}} \leq C\|\nabla v\|_{C_{*}^{0}}$. We now combine (17) with (16) to conclude that

$$
\left\|\left[\partial_{j} R\left(\left(I d-\Delta_{-1}\right) v_{j}, \cdot\right), \Delta_{q}\right] \omega\right\|_{L^{p}} \leq C(\sigma)\|\nabla v\|_{C_{*}^{0}} 2^{-q \sigma}\|\omega\|_{B_{p, \infty}^{\sigma}} .
$$

We now estimate $\left\|\left[\partial_{j} R\left(\Delta_{-1} v_{j}, \cdot\right), \Delta_{q}\right] \omega\right\|_{L^{p}}$. Using the definition of the remainder operator, as well as the properties of our partition of unity, we write

$$
\begin{aligned}
{\left[\partial_{j} R\left(\Delta_{-1} v_{j}, \cdot\right), \Delta_{q}\right] \omega } & =\partial_{j} R\left(\Delta_{-1} v_{j}, \Delta_{q} \omega\right)-\Delta_{q}\left(\partial_{j} R\left(\Delta_{-1} v_{j}, \omega\right)\right) \\
& =\partial_{j}\left(\sum_{\substack{i, k \\
|i-k| \leq 1}} \Delta_{k} \Delta_{-1} v_{j} \Delta_{i} \Delta_{q} \omega\right)-\Delta_{q} \partial_{j}\left(\sum_{\substack{i, k \\
|i-k| \leq 1}} \Delta_{k} \Delta_{-1} v_{j} \Delta_{i} \omega\right) .
\end{aligned}
$$


We begin by estimating $\Delta_{q} \partial_{j}\left(\sum_{|i-k| \leq 1}^{i, k} \Delta_{k} \Delta_{-1} v_{j} \Delta_{i} \omega\right)$. We first reintroduce the sum over $j$, allowing us to use the fact that $\operatorname{div} v=0$ to move $\partial_{j}$ inside the parentheses and differentiate $\omega$. This, along with properties of our partition of unity, gives

$$
\sum_{j=1}^{2}\left\|\Delta_{q} \partial_{j}\left(\sum_{\substack{i, k \\|i-k| \leq 1}} \Delta_{k} \Delta_{-1} v_{j} \Delta_{i} \omega\right)\right\|_{L^{p}} \leq C \sum_{j=1}^{2} \sum_{l=-1}^{1} \sum_{k=-1}^{0}\left\|\Delta_{q}\left(\Delta_{k} \Delta_{-1} v_{j} \Delta_{k-l} \partial_{j} \omega\right)\right\|_{L^{p}} .
$$

Note that in the second line of (19), the Fourier support of $\Delta_{k} \Delta_{-1} v_{j} \Delta_{k-l} \partial_{j} \omega$ is contained in a ball with radius $C 2^{k}$. Therefore, the sum in the second line is 0 if $q \geq k+M$, for a constant $M$. Furthermore, $k \leq 0$. Therefore, we are only considering $q \leq M$. We then write

$$
\begin{aligned}
& \sum_{j=1}^{2} \sum_{l=-1}^{1} \sum_{k=-1}^{0}\left\|\Delta_{q}\left(\Delta_{k} \Delta_{-1} v_{j} \Delta_{k-l} \partial_{j} \omega\right)\right\|_{L^{p}} \\
& \leq C 2^{M \sigma}\|v\|_{L^{\infty}} 2^{-q \sigma}\|\omega\|_{B_{p, \infty}^{\sigma}} .
\end{aligned}
$$

To bound $\left\|\partial_{j}\left(\sum_{|i-k|<1} \Delta_{k} \Delta_{-1} v_{j} \Delta_{i} \Delta_{q} \omega\right)\right\|_{L^{p}}$, we use the fact that the Fourier transform of $\Delta_{-1} v_{j}$ is supported in the neighborhood of the origin, and we recognize that $\operatorname{div} v=0$ allows us to move $\partial_{j}$ inside the parentheses. Therefore,

$$
\begin{aligned}
& \sum_{j=1}^{2}\left\|\partial_{j}\left(\sum_{\substack{i, k \\
|i-k| \leq 1}} \Delta_{k} \Delta_{-1} v_{j} \Delta_{i} \Delta_{q} \omega\right)\right\|_{L^{p}} \\
& \leq C \sum_{j=1}^{2} \sum_{\substack{l=-1 \\
l}}^{0}\left\|\Delta_{k=-1} \Delta_{-1} v\right\|_{L^{\infty}} 2^{k-l}\left\|\Delta_{k-l} \Delta_{q} \omega\right\|_{L^{p}} \\
& \leq C|| v\left\|_{L^{\infty}} 2^{-q \sigma}|| \omega\right\|_{B_{p, \infty}^{\sigma},}
\end{aligned}
$$

where we used Bernstein's inequality and Holder's inequality to get the first inequality in (21). We now combine (19) through (21) to conclude that

$$
\left\|\left[\partial_{j} R\left(\Delta_{-1} v_{j}, \cdot\right), \Delta_{q}\right] \omega\right\|_{L^{p}} \leq C 2^{M \sigma}\|v\|_{L^{\infty}} 2^{-q \sigma}\|\omega\|_{B_{p, \infty}^{\sigma}} .
$$

Combining (12), (15), (18), and (22), we conclude that for $q \geq 4$,

$$
\begin{aligned}
& \left\|\left[v \cdot \nabla, \Delta_{q}\right] \omega\right\|_{L_{p}} \\
& \leq C\left(2^{M \sigma}+C(\sigma)\right)\left(\left\|S_{q-1} \nabla v_{j}\right\|_{L^{\infty}}+\|\nabla v\|_{C_{*}^{0}}+\|v\|_{L^{\infty}}\right) 2^{-q \sigma}\|\omega\|_{B_{p, \infty}^{\sigma}} .
\end{aligned}
$$

To complete the proof for the case $q \geq 4$, we bound $\|\nabla v\|_{C_{*}^{0}}+\|v\|_{L^{\infty}}$ by $C\left(\left\|\omega_{0}\right\|_{L^{\infty}}+\right.$ $\left.\left\|\omega_{0}\right\|_{L^{p}}\right)$ for fixed $p<2$. This completes the proof for the case $q \geq 4$.

For the case $q<4$, write:

$$
\left[v \cdot \nabla, \Delta_{q}\right] \omega=v \cdot \nabla \Delta_{q} \omega-\Delta_{q}(v \cdot \nabla \omega) .
$$

Keeping in mind that $q \leq 3$, it is easy to see that

$$
\left\|v \cdot \nabla \Delta_{q} \omega\right\|_{L^{p}} \leq C\left(\left\|\omega_{0}\right\|_{L^{p_{0}}}+\left\|\omega_{0}\right\|_{L^{\infty}}\right) 2^{-q \sigma}\|\omega\|_{B_{p, \infty}^{\sigma}},
$$


where again we used the bound $\|v\|_{L^{\infty}} \leq C\left(\left\|\omega_{0}\right\|_{L^{p_{0}}}+\left\|\omega_{0}\right\|_{L^{\infty}}\right)$ for fixed $p_{0} \in(1,2)$. We now write the second term of (23) as

$$
\Delta_{q}(v \cdot \nabla \omega)=\sum_{j=1}^{2} \Delta_{q}\left(T_{v_{j}} \partial_{j} \omega+T_{\partial_{j} \omega} v_{j}+R\left(v_{j}, \partial_{j} \omega\right)\right) .
$$

We successfully bounded the $L^{p}$ norm of the remainder term of (25) in (16), (19), and (20) of the proof for the $q \geq 4$ case (note that (16), (19), and (20) hold for all q). Therefore, we are only concerned with $\sum_{j=1}^{2} \Delta_{q}\left(T_{v_{j}} \partial_{j} \omega+T_{\partial_{j} \omega} v_{j}\right)$. Using the fact that $S_{q^{\prime}-1} v_{j} \Delta_{q^{\prime}} \partial_{j} \omega$ has Fourier support in an annulus with inner radius $C_{1} 2^{q^{\prime}}$ and outer radius $C_{2} 2^{q^{\prime}}$, and, once again, keeping in mind that $q \leq 3$, we have

$$
\begin{aligned}
\left\|\Delta_{q}\left(T_{v_{j}} \partial_{j} \omega\right)\right\|_{L^{p}} & \leq \sum_{q^{\prime}=q-4}^{q+4} C\left\|S_{q^{\prime}-1} v\right\|_{L^{\infty}} 2^{q^{\prime}}\left\|\Delta_{q^{\prime}} \omega\right\|_{L^{p}} \\
& \leq C\left(\left\|\omega_{0}\right\|_{L^{p_{0}}}+\left\|\omega_{0}\right\|_{L^{\infty}}\right) 2^{-q \sigma}\|\omega\|_{B_{p, \infty}^{\sigma}}
\end{aligned}
$$

for $p_{0} \in(1,2)$. Furthermore, since $q \leq 3$, we write

$$
\begin{aligned}
\left\|\Delta_{q}\left(T_{\partial_{j} \omega} v_{j}\right)\right\|_{L^{p}} & \leq \sum_{q^{\prime}=q-4}^{q+4}\left\|S_{q^{\prime}-1} \partial_{j} \omega \Delta_{q^{\prime}} v_{j}\right\|_{L^{p}} \\
& \leq C \sum_{q^{\prime}=q-4}^{q+4} \sum_{k=-1}^{q^{\prime}-2} 2^{-k \sigma}\|\omega\|_{B_{p, \infty}^{\sigma}}\|v\|_{L^{\infty}} \\
& \leq C 2^{3 \sigma}\left(\left\|\omega_{0}\right\|_{L^{p_{0}}}+\left\|\omega_{0}\right\|_{L^{\infty}}\right) 2^{-q \sigma}\|\omega\|_{B_{p, \infty}^{\sigma}} .
\end{aligned}
$$

This completes the proof of the $q<4$, and therefore completes the proof of the estimate for all q. We conclude that, for all q,

$$
\left\|\left[v \cdot \nabla, \Delta_{q}\right] \omega\right\|_{L_{p}} \leq C_{1}(\sigma)\left(\left\|S_{q-1} \nabla v_{j}\right\|_{L^{\infty}}+\left\|\omega_{0}\right\|_{L^{p_{0}}}+\left\|\omega_{0}\right\|_{L^{\infty}}\right) 2^{-q \sigma}\|\omega\|_{B_{p, \infty}^{\sigma}} .
$$

Note that, if we define $h(t, x)=g(t)^{-1}(x)-x$, then $h$ satisfies an equation similar to (10). We have the following:

$$
\begin{aligned}
& \partial_{t} h_{i}+v \cdot \nabla h_{i}+v_{i}=0, \\
& \left.h_{i}\right|_{t=0}=0 .
\end{aligned}
$$

Since $h_{i}$ satisfies (27), it also satisfies

$$
\begin{aligned}
& \partial_{t} \Delta_{q} h_{i}+v \cdot \nabla \Delta_{q} h_{i}=-\Delta_{q} v_{i}+\left[v \cdot \nabla, \Delta_{q}\right] h_{i}, \\
& \left.\Delta_{q} h_{i}\right|_{t=0}=0 .
\end{aligned}
$$

This motivates us to prove a similar commutator estimate with $h$ in place of $\omega$. We prove that the following estimate holds: 
Proposition 6. Let $p \in(1, \infty), \delta>0$, and $\sigma>0$ be fixed. Then there exists two positive constants $C_{1}(\sigma)$ and $C_{2}$ such that

$$
\begin{aligned}
\left\|\left[v \cdot \nabla, \Delta_{q}\right] h\right\|_{L_{p}} & \leq C_{1}(\sigma)\left(C_{2}+\left\|S_{q-1} \nabla v\right\|_{L^{\infty}}\right) 2^{-q \sigma}\|h\|_{B_{p, \infty}^{\sigma}} \\
& +\sum_{q^{\prime}=q-4}^{q+4} C(\delta) 2^{q^{\prime} \delta}\left\|\Delta_{q^{\prime}} v\right\|_{L^{p}}\|h\|_{C^{1-\delta}} .
\end{aligned}
$$

Proof. The proof of Proposition 6 is identical to the proof of Proposition 4 with $h$ in place of $\omega$ for every term except $\Delta_{q}\left(T_{\partial_{j} h} v_{j}\right)$. Therefore, we restrict our attention to this term. This portion of the proof will result in the second piece on the right-hand side in Proposition 6.

Note that, in the proof of Proposition 4, we use the assumption that $q \geq 4$ only when bounding $\left\|\Delta_{q}\left(T_{\partial_{j} \omega} v_{j}\right)\right\|_{L^{p}}$. For all other terms, $q \geq 0$ suffices. This observation, combined with the fact that we will only need to assume $q \geq 0$ to bound $\left\|\Delta_{q}\left(T_{\partial_{j}} h v_{j}\right)\right\|_{L^{p}}$, leads us to consider the cases $q=-1$ and $q \geq 0$ separately.

We first assume $q \geq 0$, and we write $\Delta_{q}\left(T_{\partial_{j}} h v_{j}\right)=\Delta_{q}\left(\sum_{q^{\prime}=1}^{\infty} S_{q^{\prime}-1} \partial_{j} h \Delta_{q^{\prime}} v_{j}\right)$. Using the fact that $S_{q^{\prime}-1} \partial_{j} h \Delta_{q^{\prime}} v_{j}$ has Fourier support in an annulus with inner radius $C_{1} 2^{q^{\prime}}$ and outer radius $C_{2} 2^{q^{\prime}}$, we apply Bernstein's inequality and Holder's inequality to get

$$
\begin{aligned}
\left\|\Delta_{q}\left(T_{\partial_{j}} h v_{j}\right)\right\|_{L^{p}} & \leq \sum_{q^{\prime}=q-4}^{q+4} \sum_{k=-1}^{q^{\prime}-2} 2^{k \delta} 2^{k(1-\delta)}\left\|\Delta_{k} h\right\|_{L^{\infty}}\left\|\Delta_{q^{\prime}} v\right\|_{L^{p}} \\
& \leq \sum_{q^{\prime}=q-4}^{q+4} C(\delta) 2^{q^{\prime} \delta}\|h\|_{C^{1-\delta}}\left\|\Delta_{q^{\prime}} v\right\|_{L^{p}} .
\end{aligned}
$$

We now consider the case $q=-1$. As in the proof of Proposition 4 when assuming $q<4$, we begin by writing:

$$
\left[v \cdot \nabla, \Delta_{q}\right] h=v \cdot \nabla \Delta_{q} h-\Delta_{q}(v \cdot \nabla h) .
$$

For the first term of (30), we use the fact that $q=-1$ to get

$$
\left\|v \cdot \nabla \Delta_{q} h\right\|_{L^{p}} \leq C\left(\left\|\omega_{0}\right\|_{L^{p_{0}}}+\left\|\omega_{0}\right\|_{L^{\infty}}\right) 2^{-q \sigma}\|h\|_{B_{p, \infty}^{\sigma}}
$$

for $p_{0} \in(1,2)$. The second term of $(30)$ can be written as

$$
\Delta_{q}(v \cdot \nabla h)=\sum_{j=1}^{2} \Delta_{q}\left(T_{v_{j}} \partial_{j} h+T_{\partial_{j} h} v_{j}+R\left(v_{j}, \partial_{j} h\right)\right) .
$$

The proof of the bound on the $L^{p}$ norm of the remainder term in (32) is identical to the proofs of (16), (19), and (20), (which hold for all q), with $h$ in place of $\omega$. Furthermore, we handled the $L^{p}$ norm of $\Delta_{q}\left(T_{\partial_{j} h} v_{j}\right)$ (see (29), which also holds for all $q)$. Therefore, in (32), we are only concerned with $\Delta_{q}\left(T_{v_{j}} \partial_{j} h\right)$. For this term, we refer the reader to the proof for $\omega$ with $q<4$, given in (26). This completes the proof of the case $q=-1$, and therefore completes the proof of the estimate for all $q$. 


\section{Main Theorem}

In this section, we prove the following theorem:

Theorem 3. Let $v_{0} \in B_{p, \infty}^{s+1}\left(\mathbb{R}^{2}\right)$, div $v_{0}=0$, and let $\omega\left(v_{0}\right)=\omega_{0} \in C_{c}\left(\mathbb{R}^{2}\right)$, where $s p \leq 2, s \in(0,2)$, and $p \in(1, \infty)$. Let $\epsilon>0$. There exists a unique solution to (1) such that $\|v(t)\|_{B_{p, \infty}^{s+1-\epsilon}}$ belongs to $L_{\text {loc }}^{\infty}\left(\mathbb{R}^{+}\right)$.

Furthermore, let $g(t, x)$ be the measure-preserving homeomorphism satisfying $\partial_{t} g(t, x)=v(t, g(t, x))$. Define $h(t, x)=g(t)^{-1}(x)-x$. Then, under the above assumptions on $v_{0}$ and $\omega_{0}$, it follows that, for fixed $\delta>0,\|h(t)\|_{B_{p, \infty}^{s+1}-\delta}$ belongs to $L_{l o c}^{\infty}\left(\mathbb{R}^{+}\right)$.

Remark. Uniqueness in Theorem 3 follows from [10].

Proof. We begin by proving the first part of the theorem. Our approach is as follows: we fix $\epsilon^{\prime}>0$ and $T>0$, and we define $\sigma(t)=s \exp \left\{-\frac{C}{s} \epsilon^{\prime} t\right\}$. We then show that $\|v(t)\|_{B_{p, \infty}^{\sigma(t)+1}} \in L^{\infty}([0, T])$, where $C$ is an absolute constant. Letting $\epsilon=s-s \exp \left\{-\frac{C}{s} \epsilon^{\prime} T\right\}$, we make $\epsilon$ as small as we would like by our choice of $\epsilon^{\prime}$.

We first prove the theorem on a sufficiently small time interval $\left[t_{0}, t\right]$. We then use a bootstrapping argument to show that the theorem holds on any finite time interval $[0, T]$. From (11) and Proposition 4, it follows that

$$
\begin{aligned}
& \left\|\Delta_{q} \omega(t)\right\|_{L^{p}} \leq\left\|\Delta_{q} \omega\left(t_{0}\right)\right\|_{L^{p}} \\
& +\int_{t_{0}}^{t} C_{1}(\sigma(\tau))\left(C_{2}+\left\|S_{q-1} \nabla v(\tau)\right\|_{L^{\infty}}\right) 2^{-q \sigma(\tau)}\|\omega(\tau)\|_{B_{p, \infty}^{\sigma(\tau)}} d \tau .
\end{aligned}
$$

We see from the proof of Proposition 4 that $C_{1}(\sigma(t))$ can be bounded by an absolute constant for all $\sigma(t) \in(0,2)$. Therefore, for the remainder of the proof, we drop the dependence of $C_{1}$ on $\sigma(t)$. We multiply both sides of the equation by $2^{q \sigma(t)}$ and take the supremum over $q$ to get

$$
\begin{aligned}
& \|\omega(t)\|_{B_{p, \infty}^{\sigma(t)}} \leq\left\|\omega\left(t_{0}\right)\right\|_{B_{p, \infty}^{\sigma\left(t_{0}\right)}} \\
& +\sup _{q}\left\{\int _ { t _ { 0 } } ^ { t } C _ { 1 } \left(C_{2}+\left\|S_{q-1} \nabla v(\tau)\right\|_{\left.\left.L^{\infty}\right) 2^{q \sigma(t)-q \sigma(\tau)}\|\omega(\tau)\|_{B_{p, \infty}^{\sigma(\tau)}} d \tau\right\} .}\right.\right.
\end{aligned}
$$

We now show that the supremum over $q$ on the right-hand side is finite. We claim that the loss of regularity in the Besov exponent, resulting in the term $2^{q(\sigma(t)-\sigma(\tau))}$, is enough to combat the growth of $\left\|S_{q-1} \nabla v(\tau)\right\|_{L^{\infty}}$.

When taking the supremum over $q$ of the time integral, we consider two cases separately: the supremum over $q \leq N$, and the supremum over $q>N$. We then use (8) to handle the supremum over $q>N$. We write

$$
\|\omega(t)\|_{B_{p, \infty}^{\sigma(t)}} \leq\left\|\omega\left(t_{0}\right)\right\|_{B_{p, \infty}^{\sigma\left(t_{0}\right)}}+I_{1}+I_{2}
$$

where

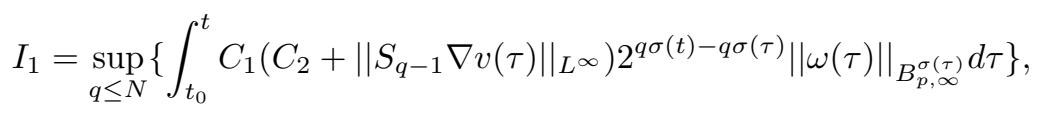


and

$$
I_{2}=\sup _{q>N}\left\{\int_{t_{0}}^{t} C_{1}\left(C_{2}+\left\|S_{q-1} \nabla v(\tau)\right\|_{L^{\infty}}\right) 2^{q \sigma(t)-q \sigma(\tau)}\|\omega(\tau)\|_{B_{p, \infty}^{\sigma(\tau)}} d \tau\right\} .
$$

To bound $I_{2}$, we apply (8), and we conclude that

$$
I_{2} \leq \int_{t_{0}}^{t} C\|\omega(\tau)\|_{B_{p, \infty}^{\sigma(\tau)}} d \tau+\sup _{q>N}\left\{\int_{t_{0}}^{t} C_{1} \epsilon q 2^{q \sigma(t)-q \sigma(\tau)}\|\omega(\tau)\|_{B_{p, \infty}^{\sigma(\tau)}} d \tau\right\} .
$$

To handle the second integral in (33), we integrate by parts. Letting $\sigma(t)=$ $s \exp \left(-\frac{2 C_{1}}{s} \epsilon t\right)$ (this is the definition of $\sigma(t)$ with $C=2 C_{1}$ ), we let $u=e^{\frac{2 C_{1}}{s} \epsilon \tau}$ and $d v=C_{1} \epsilon q e^{-\frac{2 C_{1}}{s} \epsilon \tau} 2^{q \sigma(t)-q \sigma(\tau)} d \tau$. Then, substituting $u$ and $d v$ into the second integral in (33), and recognizing that $d u$ and $v$ are positive for all $\tau \in\left[t_{0}, t\right]$, we write

$$
\begin{aligned}
\sup _{q>N}\left\{\sup _{\tau \in\left[t_{0}, t\right]}\|\omega(\tau)\|_{B_{p, \infty}^{\sigma(\tau)}} \int_{t_{0}}^{t} u d v\right\} & \leq \sup _{q>N}\left\{\sup _{\tau \in\left[t_{0}, t\right]}\|\omega(\tau)\|_{B_{p, \infty}^{\sigma(\tau)}}\left(\left.u v\right|_{t_{0}} ^{t}\right)\right\} \\
& =\sup _{\tau \in\left[t_{0}, t\right]}\|\omega(\tau)\|_{B_{p, \infty}^{\sigma(\tau)}} e^{\frac{2 C_{1}}{s} \epsilon t} \frac{1}{2 \ln 2} .
\end{aligned}
$$

We now bound the first time integral on the right-hand side of (33) by

$$
C \sup _{\tau \in\left[t_{0}, t\right]}\|\omega(\tau)\|_{B_{p, \infty}^{\sigma(\tau)}}\left(t-t_{0}\right)
$$

Combining (35) with (34), we conclude that

$$
I_{2} \leq \sup _{\tau \in\left[t_{0}, t\right]}\|\omega(\tau)\|_{B_{p, \infty}^{\sigma(\tau)}}\left\{e^{\frac{C}{s} \epsilon t} \frac{1}{2 \ln 2}+C\left(t-t_{0}\right)\right\} .
$$

To bound $I_{1}$, we first observe that $\left\|S_{q-1} \nabla v(\tau)\right\|_{L^{\infty}} \leq q\|\nabla v(\tau)\|_{C_{*}^{0}}$. Then, bounding $\|\nabla v(\tau)\|_{C_{*}^{0}}$ by $C\left(\left\|\omega_{0}\right\|_{L^{\infty}}+\left\|\omega_{0}\right\|_{L^{p_{0}}}\right)$, for $p_{0} \in(1, \infty)$, and recognizing that $2^{q(\sigma(t)-\sigma(\tau))} \leq 1$ for all $q$, we conclude that

$$
I_{1} \leq C N\left(t-t_{0}\right) \sup _{\tau \in\left[t_{0}, t\right]}\|\omega(\tau)\|_{B_{p, \infty}^{\sigma(\tau)}} .
$$

We now combine our estimates for $I_{1}$ and $I_{2}$ given in (36) and (37), which gives

$$
\sup _{\tau \in\left[t_{0}, t\right]}\|\omega(\tau)\|_{B_{p, \infty}^{\sigma(\tau)}} \leq\left\|\omega\left(t_{0}\right)\right\|_{B_{p, \infty}^{\sigma\left(t_{0}\right)}}+C^{*} \sup _{\tau \in\left[t_{0}, t\right]}\|\omega(\tau)\|_{B_{p, \infty}^{\sigma(\tau)}}
$$

where we let

$$
C^{*}=e^{\frac{C}{s} \epsilon t} \frac{1}{2 \ln 2}+C N\left(t-t_{0}\right)
$$

To complete the proof, we must make the constant $C^{*}<1$. Fix $t>0$. Given this $t$, choose $\epsilon>0$ small enough to ensure that $\frac{e^{\frac{C}{s} \epsilon t}}{2 \ln 2}<1$. Depending on our choice of $t$ and $\epsilon, N=N(t, \epsilon)$ may be very large. Given this $N$, make $t-t_{0}$ small enough so that $C^{*}<1$. Note that, under these assumptions, $C^{*}<1$ when we are working on an interval of length less than or equal to $t-t_{0}$, as long as the right endpoint of the interval is less than or equal to $t$. We therefore break $[0, t]$ into a finite number $M=M(t, \epsilon)$ of intervals of length $t-t_{0}$, and we apply a bootstrapping argument. This gives

$$
\sup _{\tau \in[0, t]}\|\omega(\tau)\|_{B_{p, \infty}^{\sigma(\tau)}} \leq C^{M}\left\|\omega_{0}\right\|_{B_{p, \infty}^{s}}
$$


where $C=\frac{1}{1-C^{*}}$, and $C^{M}$ depends on $t$ and $\epsilon$. More precisely, larger initial choice of $t$ and smaller choice of $\epsilon$ result in larger $M$ and thus larger $C^{M}$.

This completes the proof for regularity of vorticity. To show that this implies regularity of the velocity, we need the following estimate:

Lemma 7. Let $v_{0} \in B_{p, \infty}^{s+1}\left(\mathbb{R}^{2}\right)$. Then there exists two positive constants $C_{0}$ and $C_{1}$ such that

$$
\|v(t)\|_{B_{p, \infty}^{\sigma(t)+1}} \leq C_{0} e^{C_{1} t}+\|\omega(t)\|_{B_{p, \infty}^{\sigma(t)}} .
$$

Proof. We refer the reader to the proof of Lemma 6.2 in [7].

This completes the proof of the first part of the theorem.

We now prove properties of $h$. We show that $h(t) \in B_{p, \infty}^{\sigma^{\prime}(t)}\left(\mathbb{R}^{2}\right)$, where $\sigma^{\prime}(t)=$ $\sigma(t)+1-\delta$, and $\delta$ is the Holder exponent of $h(t)$ (see Theorem 2). The proof of this part of the theorem is similar to that for $\omega$. However, we must deal with the extra term which shows up in the commutator estimate given in Proposition 6.

We begin by applying Proposition 6 to (28), where, once again, we drop the dependence of $C_{1}$ on $\sigma^{\prime}$. This gives

$$
\begin{aligned}
& \left\|\Delta_{q} h(t)\right\|_{L^{p}} \leq\left\|\Delta_{q} h\left(t_{0}\right)\right\|_{L^{p}}+\int_{t_{0}}^{t} C_{1}\left(C_{2}+\left\|S_{q-1} \nabla v(\tau)\right\|_{L^{\infty}}\right) 2^{-q \sigma^{\prime}(\tau)}\|h(\tau)\|_{B_{p, \infty}^{\sigma^{\prime}(\tau)}} d \tau \\
& +\int_{t_{0}}^{t}\left\{\sum_{q^{\prime}=q-4}^{q+4}\left(\|h(\tau)\|_{C^{1-\delta}}\left\|\Delta_{q^{\prime}} v(\tau)\right\|_{L^{p}} C(\delta) 2^{q^{\prime} \delta}\right)+\left\|\Delta_{q} v(\tau)\right\|_{L^{p}}\right\} d \tau .
\end{aligned}
$$

We now multiply both sides of the inequality by $2^{q \sigma^{\prime}(t)}$ and take the supremum over $q$ to get

$$
\begin{aligned}
& \|h(t)\|\left\|_{B_{p, \infty}^{\sigma^{\prime}(t)}} \leq\right\| h\left(t_{0}\right) \|_{B_{p, \infty}^{\sigma^{\prime}\left(t_{0}\right)}} \\
& +\sup _{q}\left\{\int_{t_{0}}^{t} C_{1}\left(C_{2}+\left\|S_{q-1} \nabla v(\tau)\right\|_{\left.L^{\infty}\right)} 2^{q\left(\sigma^{\prime}(t)-\sigma^{\prime}(\tau)\right)}\|h(\tau)\|_{B_{p, \infty}^{\sigma^{\prime}(\tau)}} d \tau\right\}\right. \\
& +\int_{t_{0}}^{t}\left\{C(\delta)\|h(\tau)\|_{C^{1-\delta}}\|v(\tau)\|_{B_{p, \infty}^{\sigma(\tau)+1}}+\|v(\tau)\|_{B_{p, \infty}^{\sigma(\tau)+1}}\right\} d \tau .
\end{aligned}
$$

Here we used the fact that $\sigma^{\prime}(t)=\sigma(t)+1-\delta$, with $\delta>0$. The constant $C(\delta)$ now depends on $\sigma(\tau)$, but it is uniformly bounded for all $\sigma(\tau) \in(0,2)$.

We replace $\sigma^{\prime}(t)-\sigma^{\prime}(\tau)$ with $\sigma(t)-\sigma(\tau)$ in the first time integral, and we get

$$
\|h(t)\|_{B_{p, \infty}^{\sigma^{\prime}(t)}} \leq\left\|h\left(t_{0}\right)\right\|_{B_{p, \infty}^{\sigma^{\prime}\left(t_{0}\right)}}+J_{1}+J_{2}
$$

where

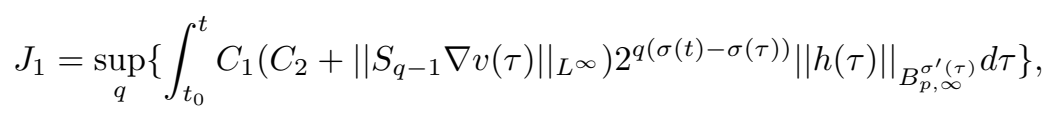

and

$$
J_{2}=\int_{t_{0}}^{t}\left\{C(\delta)\|h(\tau)\|_{C^{1-\delta}}\|v(\tau)\|_{B_{p}^{\sigma(\tau)+1}}+\|v(\tau)\|_{B_{p, \infty}^{\sigma(\tau)+1}}\right\} d \tau
$$


The argument for dealing with $J_{1}$ is identical to the argument we used to handle $I_{1}$ and $I_{2}$ when proving the first part of Theorem 3. Following this approach, we conclude that

$$
\sup _{\tau \in\left[t_{0}, t\right]}\|h(\tau)\|_{B_{p, \infty}^{\sigma^{\prime}(\tau)}} \leq\left\|h\left(t_{0}\right)\right\|_{B_{p, \infty}^{\sigma^{\prime}\left(t_{0}\right)}}+C^{*} \sup _{\tau \in\left[t_{0}, t\right]}\|h(\tau)\|_{B_{p, \infty}^{\sigma^{\prime}(\tau)}}+J_{2}
$$

where $C^{*}$ is given by (38). Arguing as we did with $\omega$, we make $C^{*}<1$ on a sufficiently short time interval and use a bootstrapping argument, as well as the fact that $h(0, x)=$ 0 , to conclude that

$$
\sup _{\tau \in[0, t]}\|h(\tau)\|_{B_{p, \infty}^{\sigma^{\prime}(\tau)}} \leq C \int_{0}^{t}\left\{C(\delta)\|h(\tau)\|_{C^{1-\delta}}\|v(\tau)\|_{B_{p, \infty}^{\sigma(\tau)+1}}+\|v(\tau)\|_{B_{p, \infty}^{\sigma(\tau)+1}}\right\} d \tau .
$$

We now observe that the right hand side of (39) is finite by Theorem 2 and by the first part of Theorem 3. This completes the proof of the theorem.

\section{Acknowledgements}

The author would like to thank Dr. Misha Vishik for his many valuable ideas and suggestions.

\section{References}

[1] H. Bahouri and J.-Y. Chemin, Équations de transport relatives á des champs de vecteurs nonlipschitziens et mécanique des fluides, Arch. Rational Mech. Anal. 127 (1994), no. 2, 159-181.

[2] J.-M. Bony, Calcul symbolique et propagation des singularités pour les équations aux dérivées partielles non linéaires, Ann. de l'Ecole Norm. Sup. 14 (1981), no. 2, 209-246.

[3] J.-Y. Chemin, Perfect incompressible fluids, Vol. 14 of Oxford Lecture Series in Mathematics and its Applications, Oxford University Press, New York (1998), ISBN 0-19-850397-0. Translated from the 1995 French original by Isabelle Gallagher and Dragos Iftimie.

[4] T. Kato and G. Ponce, Well-posedness of the Euler and Navier-Stokes equations in the Lebesgue spaces $L_{s}^{p}\left(\mathbb{R}^{2}\right)$, Rev. Mat. Iberoamericana 2 (1986), no. 1-2, 73-88.

[5] - On nonstationary flows of viscous and ideal fluids in $L_{s}^{p}\left(\mathbb{R}^{2}\right)$, Duke Math J. 55 (1987), no. $3,487-499$.

[6] H. Triebel, Theory of function spaces II, Vol. 84 of Monographs in mathematics, Birkhäuser, Basel (1992), ISBN 0817626395.

[7] M. Vishik, Hydrodynamics in Besov spaces, Arch. Ration. Mech. Anal. 145 (1998), no. 3, 197214.

[8] - Incompressible flows of an ideal fluid with vorticity in borderline spaces of Besov type, Ann. Sci. École Norm. Sup. (4) 32 (1999), no. 6, 769-812.

[9] M. Yamazaki, A quasi-homogeneous version of paradifferential operators, I. Boundedness on spaces of Besov type, J. Fac. Sci. Univ. Tokyo Sect. I A Math. 33 (1986), no. 1, 131-174.

[10] V. I. Yudovich, Non-stationary flows of an ideal incompressible fluid, Z̆. Vyčisl. Mat. i Mat. Fiz. 3 (1963) 1032-1066 (Russian).

Department of Mathematics, University of Texas, Austin, TX 78712

E-mail address: ecozzi@math.utexas.edu 